\title{
Nearest Neighbour Few-Shot Learning for Cross-lingual Classification
}

\author{
M Saiful Bari ${ }^{*} \S$, Batool Haider ${ }^{\S}$, Saab Mansour ${ }^{\S}$ \\ $\S$ Amazon AI \\ ฯ Nanyang Technological University, Singapore \\ Tbari0001@e.ntu.edu.sg \\ $\S\{$ bhaider, saabm\}@amazon.com
}

\begin{abstract}
Even though large pre-trained multilingual models (e.g. mBERT, XLM-R) have led to significant performance gains on a wide range of cross-lingual NLP tasks, success on many downstream tasks still relies on the availability of sufficient annotated data. Traditional fine-tuning of pre-trained models using only a few target samples can cause over-fitting. This can be quite limiting as most languages in the world are under-resourced. In this work, we investigate cross-lingual adaptation using a simple nearest neighbor few-shot $(<15$ samples) inference technique for classification tasks. We experiment using a total of 16 distinct languages across two NLP tasks- XNLI and PAWS-X. Our approach consistently improves traditional fine-tuning using only a handful of labeled samples in target locales. We also demonstrate its generalization capability across tasks.
\end{abstract}

\section{Introduction}

The rise of massively pre-trained multilingual language models (LM) $)^{1}$ (Lample and Conneau, 2019; Conneau et al., 2020; Chi et al., 2020; Luo et al., 2020; Xue et al., 2020) has significantly improved cross-lingual generalization across many languages (Wu and Dredze, 2019; Pires et al., 2019; K et al., 2020; Keung et al., 2019). Recent work on zeroshot cross-lingual adaptation (Bari et al., 2020; Fang et al., 2020; Pfeiffer et al., 2020), in the absence of labelled target data, has also demonstrated impressive performance gains. Despite these successes, however, there still remains a sizeable gap between supervised and zero-shot performances. On the other hand, when limited target language data are available (i.e few-shot setting), traditional fine-tuning of large pre-trained models can cause over-fitting (Perez and Wang, 2017).

\footnotetext{
${ }^{*}$ Work done while Saiful was interning at Amazon AI

${ }^{1}$ We loosely use the term LM to describe unsupervised pretrained models including Masked-LMs and Causal-LMs
}

One way to deal with the scarcity of annotated data is to augment synthetic data using techniques like paraphrasing (Gao et al., 2020; Du et al., 2020), word translation (Xie et al., 2018; Mohiuddin and Joty, 2020; Mohiuddin et al., 2020), machine translation (Sennrich et al., 2015), data-augmentation (Ding et al., 2020; Liu et al., 2021; Laskar et al., 2020; Ding et al., 2020) and/or data-diversification (Nguyen et al., 2019; Mohiuddin et al., 2021; Bari et al., 2021). Few-shot learning, on the other hand, deals with handling out-of-distribution (OOD) generalization problems using only a small amount of data (Koch, 2015; Vinyals et al., 2016; Jake Snell, 2017; Santoro et al., 2017; Chelsea Finn, 2017). In this setup, the model is evaluated over few-shot tasks, such that the model learns to generalize to new data (query set) using only a hand full of labeled samples (support set).

In a cross-lingual few-shot setup, the model learns cross-lingual features to generalize to new languages. Recently, Nooralahzadeh et al. (2020) used Meta-Learning (Finn et al., 2017) for few-shot adaptation on several cross-lingual tasks. Their fewshot setup used full development datasets of various target languages (XNLI development set, for instance, has over $2 \mathrm{~K}$ samples). In general, they showed the effectiveness of cross-lingual metatraining in the presence of a large quantity of OOD data. However, they did not provide any fine-tuning baseline. On the contrary, (Lauscher et al., 2020) explored few-shot learning but did not explore beyond fine-tuning. To the best of our knowledge, there has been no prior work in cross-lingual NLP that uses only a handful of target samples $(<15)$ and yet surpasses or matches traditional fine-tuning (on the same number of samples).

Traditional finetuning (parametric) approaches require proper hyperparameter tuning techniques for the learning rate, scheduling, optimizer, batch size, up-sampling few-shot support samples and failing to do so would often led to model over-fitting. It 
can be expensive to update parameters of large model frequently for few shot adaption, each time there is a fresh batch of support samples. As the model grows bigger, it becomes almost unscalable to update weights frequently for few shot adaptation. It takes significant amount of time to update gradients for a few number of samples and then perform inference. There have been previous successful attempts to inject external knowledge via non-parametric methods (Wang et al., 2017; Khandelwal et al., 2020, 2019).

In this work, we explore a simple Nearest Neighbor Few-shot Inference (NNFS) approach for crosslingual classification tasks. Our main objective is to utilize very few samples to perform adaptation on a given target language. To achieve this, we first fine-tune a multilingual LM on a high resource source language (i.e., English), and then apply fewshot inference using few support examples from the target language. Unlike other popular metalearning approaches that focus on improving the fine-tuning/training setup to achieve better generalization (Chelsea Finn, 2017; Ravi and Larochelle, 2017b), our approach applies to the inference phase. Hence, we do not update the weights of the LM using target language samples. This makes our approach complimentary to other regularized finetuning based few-shot meta-learning approaches. Our key contributions are as follows:

- We propose a simple method for cross-lingual few-shot adaptation on classification tasks during inference. Since our approach applies to inference, it does not require updating the LM weights using target language data.

- Using only a few labeled target support samples, we test our approach across 16 distinct languages belonging to two NLP tasks and achieve consistent improvements over traditional fine-tuning.

- We demonstrate that our proposed method generalizes well not only across languages but also across tasks.

- As the support sets are minimal in size, subsequent results obtained using them can suffer from high variability. We borrow the idea of episodic testing widely used in computer vision few-shot tasks, to evaluate few-shot performance for NLP tasks (more details in section 3.3).

We also opensource our implementation ${ }^{2}$.

\footnotetext{
${ }^{2}$ https://github.com/amazon-research/nearest-neighborcrosslingual-classification
}

\section{Method}

The objective of few-shot learning is to adapt from a source distribution to a new target distribution using only few samples. The traditional fewshot setup (Chelsea Finn, 2017; Snell et al., 2017; Vinyals et al., 2016) involves adapting a model to the distribution of new classes. Similarly, in a crosslingual setup, we adapt a pre-trained LM, that has been fine-tuned using a high resource language, to a new target language distribution (Lauscher et al., 2020; Nooralahzadeh et al., 2020).

\subsection{Setup}

We begin by fine-tuning a pre-trained model $\theta_{l m}$ (Conneau et al., 2020) to a specific task $\mathcal{T}_{s}$ using a high resource (source) language data set $\mathcal{D}_{s r c}=$ $\left(\mathcal{X}_{s r c}, \mathcal{Y}_{s r c}\right)$, to get an adapted model $\theta_{\mathcal{T}_{s}}^{\text {src }}$. We use $\theta_{\mathcal{T}_{s}}^{\text {src }}$ to perform few-shot adaptation.

In our few-shot setup, we assume to possess very few labeled support samples $\mathcal{D}_{s}=\left(\mathcal{X}_{s}, \mathcal{Y}_{s}\right)$ from the target language distribution. A support set covers $C$ classes, where each class carries $N$ number of samples. This is a standard $C$-way- $N$-shot few-shot learning setup. The objective of our proposed method is to classify the unlabeled query samples $\mathcal{D}_{q}=\left(\mathcal{X}_{q}\right)$. We denote the latent representation of the support and query samples as $X_{s}$ and $X_{q}$, respectively, where $X_{s}=\theta_{\mathcal{T}_{s}}^{\text {src }}\left(\mathcal{X}_{s}\right)$ and $X_{q}=\theta_{\mathcal{T}_{s}}^{\text {src }}\left(\mathcal{X}_{q}\right)$.

\subsection{Nearest Neighbor Class}

Let $\left|\mathcal{D}_{s}\right|$ and $\left|\mathcal{D}_{q}\right|$ be the total number of support and query samples. For query samples $\mathcal{X}_{q}$, feature representations $X_{q}$ is obtained by forward propagation on $\theta_{\mathcal{T}_{s}}^{s r c}$ model. For each query representation $x_{q}$, we define a latent binary assignment vector $y_{q}=\left[y_{q, 1}, y_{q, 2} \ldots, y_{q, C}\right]$. Here, $y_{q, i}$ is a binary variable such that,

$$
y_{q i}=\mathbb{1}_{i}\left(y_{q}\right):= \begin{cases}1 & \text { if } y_{q} \in i, \\ 0 & \text { if } y_{q} \notin i .\end{cases}
$$

and $\sum_{i} y_{q i}=1$. Let $\mathcal{Y}_{q}$ denote the $\mathcal{R}^{N_{q} \times C}$ matrix where each row represents the $y_{q}$ term of each query.

We compute the centroid, $m_{c}$, of each class by taking the mean of its support representations $\mathcal{X}_{s}$. Next, we compute the distances between each $x_{q}$ and $m_{c}$ (Equation 2). Our loss function becomes,

$$
\sum_{i=1}^{N_{q}} \sum_{c=1}^{C} y_{q, c} d\left(x_{q}, m_{c}\right)
$$


Finally, we assign each $x_{q}$ the label of the class it has the minimum distance to. This is done using the following function,

$$
y_{q c^{*}}= \begin{cases}1 & \text { if } c=\underset{\mathrm{c}^{*} \in\{1,2, \ldots, \mathrm{C}\}}{\arg \min } d\left(x_{q}, m_{c}\right) \\ 0 & \text { otherwise }\end{cases}
$$

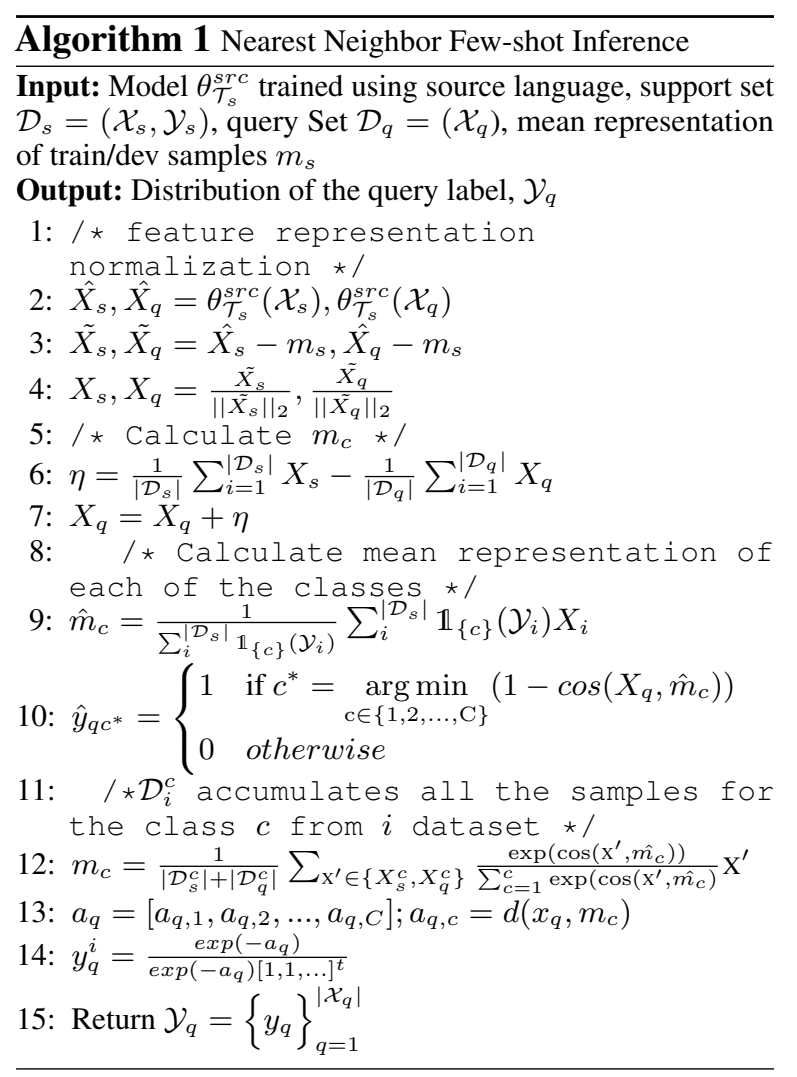

Traditional inductive inference handles each query sample (one at a time), independent of other query samples. On the contrary, our proposed approach includes additional Normalization and Transduction steps. Algorithm 1 illustrates our approach. Here we discuss these additional steps in more detail.

Norm. We measure the cross-lingual shift as the difference between the mean representations of the support set (target language) and the training set (en), $m_{s}$. We then perform cross-lingual shift correction on the query set. To achieve this, at first, we extract the latent representation of both support and query samples from $\theta_{\mathcal{T}_{s}}^{\operatorname{src}}\left(\mathcal{X}_{s}\right)$. We then center the representation (Alg 1 \#3) by subtracting the mean representation of the train/dev data of the source language, followed by L2 normalization of both representations (train/dev). Algorithm 1
(\#2-7) further details our approach.

Transduction. We apply prototypical rectification (proto-rect) (Liu et al., 2019) on the extracted features of LM. In the rectification step, to compute $m_{c}$ (in Alg.1), initially, we obtain the mean representation for each of the support classes by taking the weighted combination of $X_{s}$ and $X_{q}$. Finally, we calculate predictions on the query set using equation 3. We also present our proposed NNFS inference in Figure 2 in the Appendix.

\section{Experimental Settings}

\subsection{Data}

We use two standard multilingal datasets - $\boldsymbol{X N L I}$ (Williams et al., 2018) (15 languages) and PAWS-X (Zhang et al., 2019) (7 languages) to evaluate our proposed method. Additional details on languages and complexity of the task can be found in the Appendix. For few-Shot inference, we use samples from the target language development data to construct the support sets and the test data to construct the query sets.

\subsection{Fine-tuning}

We use XLMR-large (Conneau et al., 2020) as our pre-trained language model $\theta_{l m}$ and perform standard fine-tuning using labeled English data to adapt it to task model $\theta_{\mathcal{T}_{s}}^{s r c}$. We tune the hyper-parameters using English development data and report results using the best performing model (optimal hyperparameters have been enlisted in the appendix). We train our model using 5 different seeds and report average results across them. We use the same optimal hyper-parameters to fine-tune on the target languages. As baseline we add two additional finetuning named head and full. Fine-tuning full means all the parameters of the model are updated. This is very unlikely in Few-shot scenarios. Fine-tuning head means only the parameters of the last linear layer are updated.

\subsection{Evaluation Setup}

Nooralahzadeh et al. (2020) and Lauscher et al. (2020) used 10 and 5 different seeds to measure the few-shot performance. As few-shot learning involves randomly selecting small support sets, results may vary greatly from one experiment to the next, and hence may not be reliable (Le et al., 2020). In computer vision, episodic testing (Ravi and Larochelle, 2017a; Li et al., 2019; Ziko et al., 


\begin{tabular}{lcccccccccccccccc}
\hline Exp. Type & Resource & fr & es & de & el & bg & ru & tr & ar & vi & th & zh & hi & sw & ur & avg \\
\hline \hline & & \multicolumn{8}{c}{$\theta_{\mathcal{T}_{s}}^{\text {src }}=$ Finetuned-XLM-R-large } & with XNLI dataset & & & & & & \\
\hline \hline Zero-Shot & en & 83.1 & 84.8 & 83.0 & 82.2 & 83.4 & 80.1 & 78.8 & 78.8 & 80.1 & 78.1 & 79.4 & 76.7 & 72.7 & 72.9 & 79.6 \\
NN & en+fs-3.5 & 83.0 & 84.6 & 82.7 & 82.0 & 83.3 & 80.3 & 78.9 & 79.2 & 80.2 & 78.3 & 79.5 & 76.6 & 71.9 & 73.0 & 79.5 \\
+proto-rect & en+fs-3.5 & 83.7 & 85.2 & $\mathbf{8 3 . 5}$ & 82.7 & 84.1 & 81.2 & $\mathbf{7 9 . 8}$ & $\mathbf{8 0 . 3}$ & 81.2 & 79.4 & $\mathbf{8 0 . 4}$ & $\mathbf{7 7 . 7}$ & $\mathbf{7 3 . 5}$ & $\mathbf{7 4 . 4}$ & $\mathbf{8 0 . 5}$ \\
+norm & en+fs-3.5 & 83.1 & 84.6 & 82.8 & 82.1 & 83.5 & 80.4 & 79.0 & 79.3 & 80.4 & 78.5 & 79.6 & 76.6 & 71.8 & 73.0 & 79.6 \\
+proto-rect & en+fs-3.5 & $\mathbf{8 3 . 8}$ & $\mathbf{8 5 . 2}$ & 83.4 & $\mathbf{8 2 . 8}$ & $\mathbf{8 4 . 2}$ & $\mathbf{8 1 . 3}$ & $\mathbf{7 9 . 8}$ & 80.2 & $\mathbf{8 1 . 3}$ & $\mathbf{7 9 . 4}$ & 80.3 & $\mathbf{7 7 . 7}$ & 73.2 & 74.2 & $\mathbf{8 0 . 5}$ \\
Fine-tuning (full) & en+fs-3.5 & 83.2 & 84.6 & 82.9 & 82.2 & 83.5 & 80.8 & 79.2 & 79.5 & 80.5 & 78.6 & 80.2 & 77.0 & 72.6 & 74.0 & 79.9 \\
Fine-tuning (head) & en+fs-3.5 & 83.2 & 84.9 & 83.2 & 82.3 & 83.5 & 80.4 & 79.0 & 79.1 & 80.3 & 78.4 & 79.6 & 76.9 & 72.9 & 73.2 & 79.8 \\
\hline
\end{tabular}

Table 1: Few-shot XNLI accuracy results across 14 languages with average improvements for each of the methods. All the confidence interval is less than .07 in the experiments. "fs-3.5" means 3-way-5-shot learning.

\begin{tabular}{|c|c|c|c|c|c|c|c|c|}
\hline Exp. Type & Resource & de & es & fr & ja & ko & $\mathbf{z h}$ & avg \\
\hline \multicolumn{9}{|c|}{$\theta_{\mathcal{T}_{s}}^{s T c}=$ Finetuned-XLM-R-large with PAWS-X dataset } \\
\hline Zero-Shot & en & 89.8 & 89.6 & 90.5 & 78.8 & 78.6 & 81.9 & 84.9 \\
\hline $\mathrm{NN}$ & en+fs-2.5 & 89.8 & 89.8 & 90.6 & 79.8 & 80.4 & 82.5 & 85.5 \\
\hline +proto-rect & en+fs-2.5 & 90.3 & 90.2 & 91.0 & 80.5 & 81.2 & 83.3 & 86.1 \\
\hline +norm & $e n+f s-2.5$ & 90.0 & 90.2 & 90.8 & 79.9 & 80.7 & 82.7 & 85.7 \\
\hline +proto-rect & $\mathrm{en}+\mathrm{fs}-2.5$ & 90.4 & 90.6 & 91.2 & 80.5 & 81.3 & 83.5 & 86.3 \\
\hline Fine-tuning (full) & $\mathrm{en}+\mathrm{fs}-2.5$ & 88.9 & 89.1 & 89.6 & 79.2 & 79.7 & 82.0 & 84.7 \\
\hline Fine-tuning (head) & en+fs- 2.5 & 90.0 & 89.8 & 90.7 & 79.3 & 79.5 & 82.1 & 85.3 \\
\hline
\end{tabular}

Table 2: Few-shot PAWS-X acc. results across 6 languages. Here in Resource column, "en" indicates model is trained with full English training data. $f s-2.5$ means 2-way-5-shot learning.

\begin{tabular}{|c|c|c|c|c|c|c|c|c|c|}
\hline Exp. Type & Resource & en & de & es & fr & $\mathbf{j a}$ & ko & zh & avg \\
\hline \multicolumn{10}{|c|}{$\theta_{\mathcal{T}_{s}}^{s r c}=$ Finetuned-XLM-R-large with XNLI dataset } \\
\hline Zero-Shot & & 41.4 & 43.5 & & & 46.0 & & & 44.3 \\
\hline NN & & 71 & 66 & & & & & & 64.4 \\
\hline +proto-re & & 70.5 & 66.1 & 65.1 & 66.2 & 60.0 & & & 64.0 \\
\hline & & 72.2 & 67.8 & 66.1 & 67.2 & 60.8 & & & 65.2 \\
\hline & & 72 & & & & & & & 65.0 \\
\hline Fine-t & & 64 & $5 \mathrm{~s}$ & 58 & & & & 4.8 & 57.7 \\
\hline Fine-tuning (head) & en+fs- 2.5 & 48.2 & 47.9 & 48.3 & 48.2 & 47.7 & 48.2 & 46.8 & 47.9 \\
\hline
\end{tabular}

Table 3: PAWS-X accuracy results for cross-task experiments across 6 languages. For this experiment, we fine-tuned XLM-R LM using the XNLI task and then applied few-shot inference on the PAWS-X task.

2020) is often used for evaluating few-shot experiments. Each episode is composed of small randomly selected support and query sets. Model's performance on each episode is noted, and the average performance score, alongside the confidence interval (95\%) across all episodes are reported. To the best of our knowledge, episodic testing has not been leveraged for cross-lingual few-shot learning in NLP.

We evaluate our approach using 300 episodes per seed model $\theta_{\mathcal{T}_{s}}^{\text {src }}$ totalling 1500 episodic testing and report their average scores. For each episode, we perform C-way-N-shot inference. For 2-way-5-shot setting, for instance, we randomly select 15 query samples per class, and $2 \times 5$ number of support samples. For XNLI and PAWS-X, we use 3 and 2 as the value of $\mathrm{C}$, respectively. Our episodic testing approach has been detailed further in the Episodic Algorithm of the Appendix.

\subsection{Results and Analysis}

After training the model with the source language samples (i.e. labeled English data), we perform additional fine-tuning using $C$-way-5-shot target language samples. Finally, we perform our proposed NNFS inference.

The fine-tuning baseline using limited target language samples result in small but non-significant improvements over the zero-shot baseline. The NNFS inference approach, however, resulted in performance gains using only 15 (3-way-5-shot) and 10 (2-way-5-shot) support examples for both XNLI and PAWS-X tasks. When compared to the few-shot baseline, we got an average improvement of 0.6 on XNLI (table 4) and 1.0 on PAWS-X (table 5). At first we experimented with 3-shot support samples but did not observe any few-shot capability in the model. We also experimented with 10-shot setup and found similar improvements of NNFS on top of the Fine-tuning baseline (results have been added to the Appendix). Interestingly, for both cases, we observed higher performance gains on low resource languages.

To further evaluate the effectiveness of our model, we tested it in a cross-task setting. We first trained the model on XNLI (EN data) and then used NNFS inference on PAWS-X. Table 3 demonstrates an impressive average performance gain of +7.3 across all PAWS-X languages, over the fine-tuning baseline.

In addition to that, NNFS inference approach is fast. When compared to the zero-shot inference $(1 \mathrm{x})$, our approach takes only $\approx 1.36-1.7 \mathrm{x}$ time of computation cost compared to the finetuning time which takes $\approx 38-40 \mathrm{x}$. Table 6 in appendix shows the inference time details on both tasks. 


\section{Conclusion}

The paper proposes a nearest neighbour based fewshot adaptation algorithm accompanied by a necessary evaluation protocol. Our approach does not require updating the LM weights and thus avoids over-fitting to limited samples. We experiment using two classification tasks and results demonstrate consistent improvements over finetuning not only across languages, but also across tasks.

\section{References}

M Saiful Bari, Shafiq Joty, and Prathyusha Jwalapuram. 2020. Zero-Resource Cross-Lingual Named Entity Recognition. In Proceedings of the 34th AAAI Conference on Artifical Intelligence, AAAI '20, pages $\mathrm{xx}-\mathrm{xx}$, New York, USA. AAAI.

M Saiful Bari, Tasnim Mohiuddin, and Shafiq Joty. 2021. UXLA: A robust unsupervised data augmentation framework for zero-resource cross-lingual NLP. In Proceedings of the 59th Annual Meeting of the Association for Computational Linguistics and the 11th International Joint Conference on Natural Language Processing (Volume 1: Long Papers), pages 1978-1992, Online. Association for Computational Linguistics.

Sergey Levine Chelsea Finn, Pieter Abbeel. 2017. Model-agnostic meta-learning for fast adaptation of deep networks.

Zewen Chi, Li Dong, Furu Wei, Nan Yang, Saksham Singhal, Wenhui Wang, Xia Song, Xian-Ling Mao, Heyan Huang, and Ming Zhou. 2020. Infoxlm: An information-theoretic framework for cross-lingual language model pre-training.

Alexis Conneau, Kartikay Khandelwal, Naman Goyal, Vishrav Chaudhary, Guillaume Wenzek, Francisco Guzmán, Edouard Grave, Myle Ott, Luke Zettlemoyer, and Veselin Stoyanov. 2020. Unsupervised cross-lingual representation learning at scale. In Proceedings of the 58th Annual Meeting of the Association for Computational Linguistics, pages 8440 8451, Online. Association for Computational Linguistics.

Alexis Conneau, Guillaume Lample, Ruty Rinott, Adina Williams, Samuel R. Bowman, Holger Schwenk, and Veselin Stoyanov. 2018. XNLI: evaluating cross-lingual sentence representations. CoRR, abs/1809.05053.

Bosheng Ding, Linlin Liu, Lidong Bing, Canasai Kruengkrai, Thien Hai Nguyen, Shafiq Joty, Luo Si, and Chunyan Miao. 2020. DAGA: Data augmentation with a generation approach for low-resource tagging tasks. In Proceedings of the 2020 Conference on Empirical Methods in Natural Language Processing (EMNLP), pages 6045-6057, Online. Association for Computational Linguistics.
Jingfei Du, Edouard Grave, Beliz Gunel, Vishrav Chaudhary, Onur Celebi, Michael Auli, Ves Stoyanov, and Alexis Conneau. 2020. Self-training improves pre-training for natural language understanding. CoRR, abs/2010.02194.

Yuwei Fang, Shuohang Wang, Zhe Gan, Siqi Sun, and Jingjing Liu. 2020. Filter: An enhanced fusion method for cross-lingual language understanding.

Chelsea Finn, Pieter Abbeel, and Sergey Levine. 2017. Model-agnostic meta-learning for fast adaptation of deep networks. CoRR, abs/1703.03400.

Silin Gao, Yichi Zhang, Zhijian Ou, and Zhou Yu. 2020. Paraphrase augmented task-oriented dialog generation.

Richard S. Zemel Jake Snell, Kevin Swersky. 2017. Prototypical networks for few-shot learning.

Karthikeyan K, Zihan Wang, Stephen Mayhew, and Dan Roth. 2020. Cross-lingual ability of multilingual $\{$ bert $\}$ : An empirical study. In International Conference on Learning Representations.

Phillip Keung, yichao lu, and Vikas Bhardwaj. 2019. Adversarial learning with contextual embeddings for zero-resource cross-lingual classification and ner. Proceedings of the 2019 Conference on Empirical Methods in Natural Language Processing and the 9th International Joint Conference on Natural Language Processing (EMNLP-IJCNLP).

Urvashi Khandelwal, Angela Fan, Dan Jurafsky, Luke Zettlemoyer, and Mike Lewis. 2020. Nearest neighbor machine translation. CoRR, abs/2010.00710.

Urvashi Khandelwal, Omer Levy, Dan Jurafsky, Luke Zettlemoyer, and Mike Lewis. 2019. Generalization through memorization: Nearest neighbor language models. CoRR, abs/1911.00172.

Gregory R. Koch. 2015. Siamese neural networks for one-shot image recognition.

Guillaume Lample and Alexis Conneau. 2019. Crosslingual language model pretraining. Advances in Neural Information Processing Systems (NeurIPS).

Md. Tahmid Rahman Laskar, Enamul Hoque, and Jimmy Xiangji Huang. 2020. WSL-DS: weakly supervised learning with distant supervision for query focused multi-document abstractive summarization. CoRR, abs/2011.01421.

Anne Lauscher, Vinit Ravishankar, Ivan Vulić, and Goran Glavaš. 2020. From zero to hero: On the limitations of zero-shot cross-lingual transfer with multilingual transformers.

Canyu Le, Zhonggui Chen, Xihan Wei, Biao Wang, and Lei Zhang. 2020. Continual local replacement for few-shot learning. 
Hongyang Li, David Eigen, Samuel Dodge, Matthew Zeiler, and Xiaogang Wang. 2019. Finding taskrelevant features for few-shot learning by category traversal. CoRR, abs/1905.11116.

Jinlu Liu, Liang Song, and Yongqiang Qin. 2019. Prototype rectification for few-shot learning. CoRR, abs/1911.10713.

Linlin Liu, Bosheng Ding, Lidong Bing, Shafiq Joty, Luo Si, and Chunyan Miao. 2021. MulDA: A multilingual data augmentation framework for lowresource cross-lingual NER. In Proceedings of the 59th Annual Meeting of the Association for Computational Linguistics and the 11th International Joint Conference on Natural Language Processing (Volume 1: Long Papers), pages 5834-5846, Online. Association for Computational Linguistics.

Fuli Luo, Wei Wang, Jiahao Liu, Yijia Liu, Bin Bi, Songfang Huang, Fei Huang, and Luo Si. 2020. Veco: Variable encoder-decoder pre-training for cross-lingual understanding and generation.

Tasnim Mohiuddin, M Saiful Bari, and Shafiq Joty. 2020. LNMap: Departures from isomorphic assumption in bilingual lexicon induction through nonlinear mapping in latent space. In Proceedings of the 2020 Conference on Empirical Methods in Natural Language Processing (EMNLP), pages 2712-2723, Online. Association for Computational Linguistics.

Tasnim Mohiuddin, M. Saiful Bari, and Shafiq R. Joty. 2021. AUGVIC: exploiting bitext vicinity for lowresource NMT. CoRR, abs/2106.05141.

Tasnim Mohiuddin and Shafiq Joty. 2020. Unsupervised Word Translation with Adversarial Autoencoder. Computational Linguistics, 46(2):257-288.

Xuan-Phi Nguyen, Shafiq R. Joty, Wu Kui, and Ai Ti Aw. 2019. Data diversification: An elegant strategy for neural machine translation. CoRR, abs/1911.01986.

Farhad Nooralahzadeh, Giannis Bekoulis, Johannes Bjerva, and Isabelle Augenstein. 2020. Zero-shot cross-lingual transfer with meta learning.

Luis Perez and Jason Wang. 2017. The effectiveness of data augmentation in image classification using deep learning. CoRR, abs/1712.04621.

Jonas Pfeiffer, Ivan Vulić, Iryna Gurevych, and Sebastian Ruder. 2020. Mad-x: An adapter-based framework for multi-task cross-lingual transfer.

Telmo Pires, Eva Schlinger, and Dan Garrette. 2019. How multilingual is multilingual BERT? In Proceedings of the 57th Annual Meeting of the Association for Computational Linguistics, pages 49965001, Florence, Italy. Association for Computational Linguistics.

S. Ravi and H. Larochelle. 2017a. Optimization as a model for few-shot learning. In ICLR.
Sachin Ravi and Hugo Larochelle. 2017b. Optimization as a model for few-shot learning. ICLR, abs/1703.03400.

Adam Santoro, David Raposo, David G. T. Barrett, Mateusz Malinowski, Razvan Pascanu, Peter W. Battaglia, and Timothy P. Lillicrap. 2017. A simple neural network module for relational reasoning. CoRR, abs/1706.01427.

Rico Sennrich, Barry Haddow, and Alexandra Birch. 2015. Improving neural machine translation models with monolingual data. CoRR, abs/1511.06709.

Jake Snell, Kevin Swersky, and Richard S. Zemel. 2017. Prototypical networks for few-shot learning. CoRR, abs/1703.05175.

Oriol Vinyals, Charles Blundell, Timothy P. Lillicrap, Koray Kavukcuoglu, and Daan Wierstra. 2016. Matching networks for one shot learning. CoRR, abs/1606.04080.

Zhiguo Wang, Wael Hamza, and Linfeng Song. 2017. $\mathrm{k}$-nearest neighbor augmented neural networks for text classification. CoRR, abs/1708.07863.

Adina Williams, Nikita Nangia, and Samuel Bowman. 2018. A broad-coverage challenge corpus for sentence understanding through inference. In Proceedings of the 2018 Conference of the North American Chapter of the Association for Computational Linguistics: Human Language Technologies, Volume 1 (Long Papers), pages 1112-1122. Association for Computational Linguistics.

Shijie Wu and Mark Dredze. 2019. Beto, bentz, becas: The surprising cross-lingual effectiveness of BERT. In Proceedings of the 2019 Conference on Empirical Methods in Natural Language Processing and the 9th International Joint Conference on Natural Language Processing (EMNLP-IJCNLP), pages 833-844, Hong Kong, China. Association for Computational Linguistics.

Jiateng Xie, Zhilin Yang, Graham Neubig, Noah A. Smith, and Jaime Carbonell. 2018. Neural crosslingual named entity recognition with minimal resources. In Proceedings of the 2018 Conference on Empirical Methods in Natural Language Processing, pages 369-379, Brussels, Belgium. Association for Computational Linguistics.

Linting Xue, Noah Constant, Adam Roberts, Mihir Kale, Rami Al-Rfou, Aditya Siddhant, Aditya Barua, and Colin Raffel. 2020. mt5: A massively multilingual pre-trained text-to-text transformer.

Yuan Zhang, Jason Baldridge, and Luheng He. 2019. PAWS: Paraphrase adversaries from word scrambling. In Proceedings of the 2019 Conference of the North American Chapter of the Association for Computational Linguistics: Human Language Technologies, Volume 1 (Long and Short Papers), pages 1298-1308, Minneapolis, Minnesota. Association for Computational Linguistics. 
Imtiaz Masud Ziko, Jose Dolz, Eric Granger, and Ismail Ben Ayed. 2020. Laplacian regularized fewshot learning.

\section{A Appendices}

\section{A.1 Decision choice for Episodic Testing}

In the traditional testing framework, we sample a batch from the dataset and calculate the batch's prediction. Finally, accumulate all the predictions to calculate the score of the evaluation metric. However, Few-shot experiments are quite unpredictable because of the following two reasons,

- Support set: Per class sampling strategy of the support set is random. In a few shot experiments, we perform inference on the test dataset utilizing support-samples. For a different support set, the prediction may vary drastically. However, taking few samples (ie., 10 out of 2500 or 15 out of 2000) and doing experiments 5-10 times doesn't reflect the true potential of a few-shot algorithm.

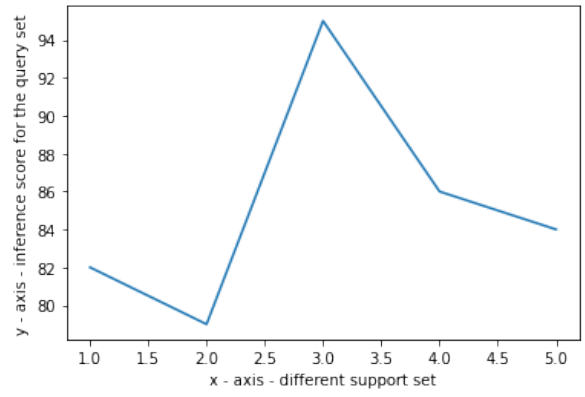

Figure 1: For a same query set result varies because of different support set.

- Transductive inference: On the contrary, for a few shot experiments, algorithms often perform transductive inference. In transductive inference, predictions may vary based on the combination of the query samples. Hence it is challenging to benchmark the few shot algorithms with the traditional testing framework.

In Episodic testing, we randomly sample a query set and support set from the dataset and perform few-shot experiments. We perform the experiments until we get a low confidence-interval (95\%). In this way, we may iterate over the test dataset 510 times more. However, it is not affected by the above problems mentioned and can benchmark any few-shot algorithm properly.

\section{A.2 Extended Dataset}

XNLI We use XNLI dataset (Conneau et al., 2018) which extends the MultiNLI dataset (Williams et al., 2018) to 15 languages. MultiNLI dataset contains sentences from 10 different genres. The objective is to identify if a premise entails with the hypothesis. It is a crowd sourced 3-class classification dataset covering 14 languages that have been translated from English. These locales include French (fr), Spanish (es), German (de), Greek (el), Bulgarian (bg), Russian (ru), Turkish (tr), Arabic (a), Vietnamese (vi), Thai (th), Chinese (zh), Hindi (hi), Swahili (sw), and Urdu (ur). It comes with human translated dev and test splits. The dataset is balanced and contains 392702, 2490 and 5010 numbers of train, dev and test instance for each of the language, respectively.

PAWSX Given a pair of sentences, the objective of PAWS (Paraphrase Adversaries from Word Scrambling) (Zhang et al., 2019) is to classify if the pair is a paraphrase or not. PAWS-X dataset contains six topologically different languages that have been machine translated from English. These include French (fr), Spanish (es), German (de), Korean (ko), Japanese (ja), and Chinese (zh). Similar to XNLI, it also comes with human translated dev and test split.

Challenges Both datasets posses different challenges. NLI task requires rich and a high level of factual understanding of the text. The PAWS task, on the other hand, contains pairs of sentences that usually have a high lexical overlap and may/may not be paraphrases. We use accuracy as the evaluation metric for both datasets.

10 Shot results For reference we have added 10 shot experiment for XNLI and PAWSX dataset with same setup as Table 1 and Table 2 of main paper.

\section{A.3 Hyperparameters and Resource Description}

We used 8 V100 GPUs (amazon p3.16xlarge) to run all experiments. The hyper-parameters of the best performing model are enlisted in Table 6 . In the pretrained language model finetuning, We use (1e-5, 3e-5, 5e-5, 7.5e-6,5e-6) boundary values to search for proper learning rate. 


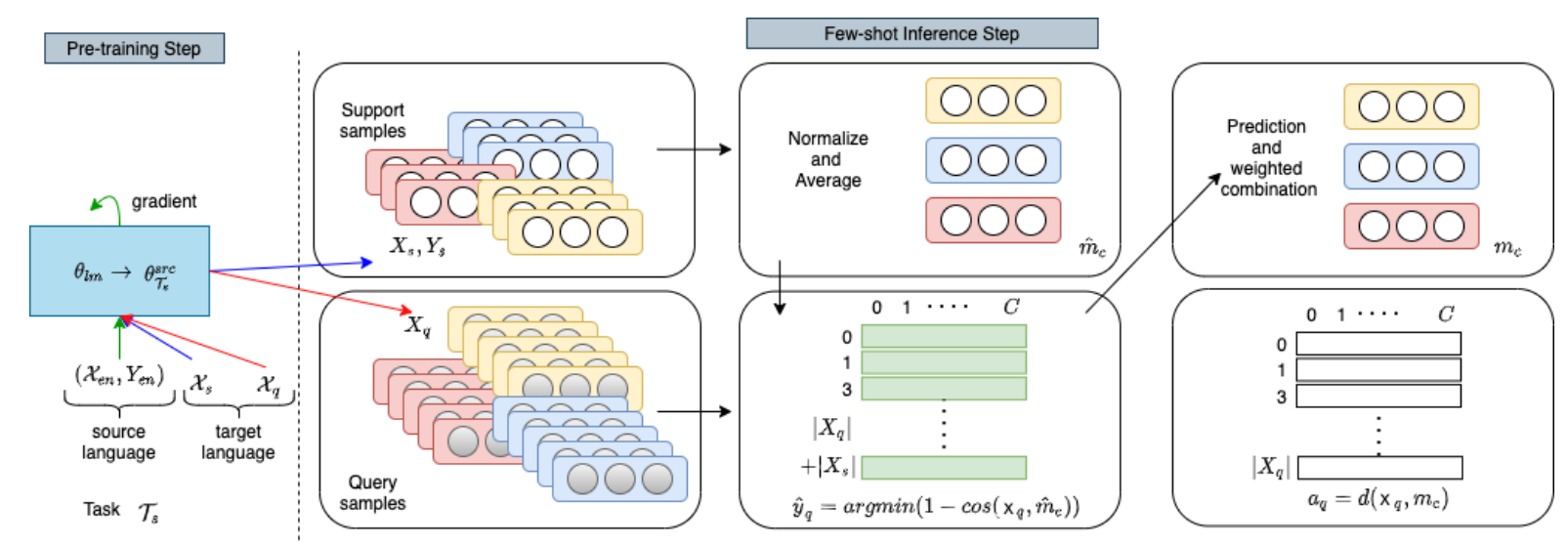

Figure 2: Training flow diagram of nearest neighbour few-shot learning for cross-lingual NLP. In the Pre-training Step we train a language model $\theta_{l m}$ on the source language (en) data $\left(\mathcal{X}_{s r c}, \mathcal{Y}_{s r c}\right)$ to get $\theta_{\mathcal{T}_{s}}^{s r c}$ In Few-Shot Inference Step, we apply forward propagation on the $\theta_{\mathcal{T}_{s}}^{\text {src }}$ model using support input samples $\mathcal{X}_{s}^{s}$ and $\mathcal{X}_{q}$ and get the latent representations $X_{s}$ and $Y_{s}$. Using $X_{s}$, we apply normalization and calculate $m_{c}$. We then use both $X_{s}$ and $X_{q}$, and compute the unary term $a_{q}$, which in turn gives the label distribution of the query samples (see in Alg. Few Shot Inference. line \#14-15 ).

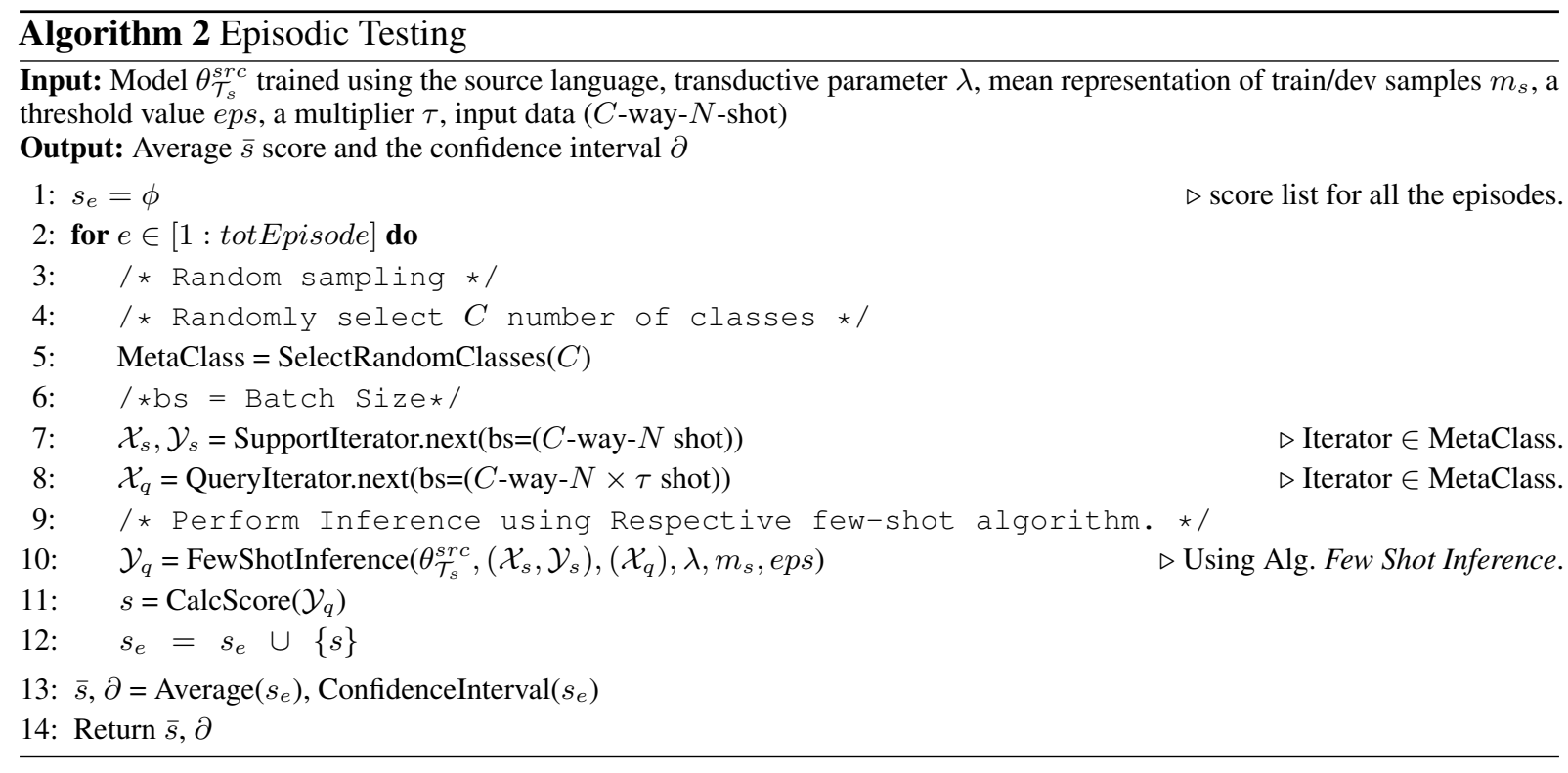

\begin{tabular}{lcccccccccccccccc}
\hline Exp. Type & Resource & fr & es & de & el & bg & ru & tr & ar & vi & th & zh & hi & sw & ur & avg \\
\hline \hline & & \multicolumn{1}{c}{$\theta_{\mathcal{T}_{s}}^{\text {src }}=$ Finetuned-XLM-R-large } & with XNLI dataset & & & & & & \\
\hline \hline Zero-Shot & en & 83.1 & 84.8 & 83.0 & 82.1 & 83.3 & 80.2 & 78.9 & 78.7 & 80.1 & 78.1 & 79.5 & 76.7 & 72.5 & 73.0 & 79.6 \\
NN & en+fs-3.10 & 83.4 & 85.0 & 83.1 & 82.5 & 83.8 & 80.9 & 79.5 & 79.8 & 80.8 & 79.2 & 80.3 & 77.4 & 72.9 & 74.0 & 80.2 \\
+proto-rect & en+fs-3.10 & $\mathbf{8 3 . 8}$ & $\mathbf{8 5 . 3}$ & $\mathbf{8 3 . 6}$ & $\mathbf{8 2 . 8}$ & 84.1 & 81.2 & 79.9 & $\mathbf{8 0 . 4}$ & 81.3 & 79.6 & $\mathbf{8 0 . 7}$ & $\mathbf{7 8 . 1}$ & $\mathbf{7 3 . 6}$ & $\mathbf{7 4 . 7}$ & 80.6 \\
+norm & en+fs-3.10 & 83.5 & 85.0 & 83.2 & 82.6 & 83.8 & 81.1 & 79.6 & 79.8 & 81.0 & 79.3 & 80.3 & 77.5 & 73.0 & 74.0 & 80.3 \\
+norm+proto-rect & en+fs-3.10 & $\mathbf{8 3 . 8}$ & 85.2 & $\mathbf{8 3 . 6}$ & $\mathbf{8 2 . 8}$ & $\mathbf{8 4 . 2}$ & $\mathbf{8 1 . 4}$ & $\mathbf{8 0 . 0}$ & $\mathbf{8 0 . 4}$ & $\mathbf{8 1 . 4}$ & $\mathbf{7 9 . 7}$ & $\mathbf{8 0 . 7}$ & $\mathbf{7 8 . 1}$ & 73.5 & 74.6 & $\mathbf{8 0 . 7}$ \\
Fine-tuning (full) & en+fs-3.10 & 83.2 & 84.5 & 82.9 & 82.5 & 83.7 & 81.2 & 79.5 & 79.8 & 80.8 & 78.9 & 80.5 & 77.3 & 72.6 & 74.2 & 80.1 \\
Fine-tuning (head) & en+fs-3.10 & 83.3 & 85.0 & 83.2 & 82.4 & 83.5 & 80.6 & 79.2 & 79.4 & 80.5 & 78.6 & 79.9 & 77.2 & 72.8 & 73.6 & 79.9 \\
\hline
\end{tabular}

Table 4: 10-shot XNLI accuracy results across 14 languages with average improvements for each of the methods. All the confidence interval is less than .07 in the experiments.

\section{B Reproducibility Settings and Notes}

- python $_{3.6 .13} . \quad$ Pytorch $_{1.7 .1}, \quad C U D A_{10.2}$,
- transformers 4.6 .0

- Average runtime: See table 7. cuDNN $N_{7605}$ 


\begin{tabular}{lccccccccc}
\hline Exp. Type & Resource & de & es & fr & ja & ko & zh & avg \\
\hline \hline \multicolumn{2}{l}{$\theta_{\mathcal{T}_{s}}^{\text {src }}$} & = & Finetuned-XLM-R-large & with PAWS-X dataset & & \\
\hline \hline Zero-Shot & en & 89.8 & 89.6 & 90.6 & 78.8 & 78.4 & 81.8 & 84.8 \\
NN & en+fs-2.10 & 90.0 & 90.1 & 90.8 & 80.2 & 80.7 & 83.2 & 85.8 \\
+proto-rect & en+fs-2.10 & 90.3 & 90.3 & 91.2 & 80.5 & 81.2 & 83.5 & 86.2 \\
+norm & en+fs-2.10 & 90.1 & 90.4 & 91.1 & 80.3 & 81.0 & 83.3 & 86.0 \\
+norm+proto-rect & en+fs-2.10 & $\mathbf{9 0 . 4}$ & $\mathbf{9 0 . 7}$ & $\mathbf{9 1 . 4}$ & $\mathbf{8 0 . 7}$ & $\mathbf{8 1 . 5}$ & $\mathbf{8 3 . 7}$ & $\mathbf{8 6 . 4}$ \\
Fine-tuning (full) & en+fs-2.10 & 89.4 & 89.6 & 90.1 & 79.9 & 80.6 & 82.6 & 85.4 \\
Fine-tuning (head) & een+fs-2.10 & 90.1 & 90.1 & 91.0 & 79.9 & 79.8 & 82.5 & 85.6 \\
\hline
\end{tabular}

Table 5: 10-shot PAWS-X acc. results across 6 languages. Here in Resource column, "en" indicates model is trained with full english training data.

\begin{tabular}{l|c}
\hline Hyperparameter & Value \\
\hline LM & XLMR-large \\
\# of params & $550 \mathrm{M}$ \\
learning rate & $7.5 \mathrm{e}-6$ \\
Max Sequence Length & 128 \\
Per GPU batch size & 8 \\
Gradient accumulation step & 2 \\
Multi-GPU training & 8 \\
Effective batch size & 128 \\
Number of epoch & 10 \\
Warmup step in pre-training & $6 \%$ of total number of steps \\
Total number of episodic test & 1000 \\
finetuning batch-size & 16 \\
finetuning learning rate & $7.5 \mathrm{e}-6$ \\
finetuning schedueler & constant scheduler \\
\hline
\end{tabular}

Table 6: Optimal hyper-parameter settings.

\begin{tabular}{lcccc}
\hline \multirow{2}{*}{ Exp. Type } & \multicolumn{2}{c}{ PAWSX } & \multicolumn{2}{c}{ XNLI } \\
& fs-2.5 & fs-2.10 & fs-3.5 & fs-3.10 \\
\hline Zero-Shot & $1 \mathrm{x}$ & $1 \mathrm{x}$ & $1.35 \mathrm{x}$ & $1 \mathrm{x}$ \\
NN & $1.36 \mathrm{x}$ & $1.71 \mathrm{x}$ & $1.35 \mathrm{x}$ & $1.66 \mathrm{x}$ \\
$\quad$ +proto-rect & $1.37 \mathrm{x}$ & $1.71 \mathrm{x}$ & $1.35 \mathrm{x}$ & $1.67 \mathrm{x}$ \\
$\quad$ +norm & $1.36 \mathrm{x}$ & $1.71 \mathrm{x}$ & $1.35 \mathrm{x}$ & $1.66 \mathrm{x}$ \\
$\quad$ +proto-rect & $\mathbf{1 . 3 7 x}$ & $\mathbf{1 . 7 1 x}$ & $\mathbf{1 . 3 5 x}$ & $\mathbf{1 . 6 7 x}$ \\
Fine-tuning (full) & $22.44 \mathrm{x}$ & $41.86 \mathrm{x}$ & $21.01 \mathrm{x}$ & 38.69 \\
Fine-tuning (head) & $20.48 \mathrm{x}$ & $38.02 \mathrm{x}$ & $19.24 \mathrm{x}$ & 35.17 \\
\hline
\end{tabular}

Table 7: Inference time for each of the task. 